\title{
Acute acetaminophene-induced hepatotoxicity and nephrotoxicity; therapeutic effect of dexmedetomidine
}

\author{
Tas $\mathrm{N}^{1}$, Altinbas $\mathrm{A}^{2}$, Noyan $\mathrm{T}^{3}$, Kokturk $\mathrm{S}^{4}$, Ayhan $\mathrm{S}^{3}$, Canakci $\mathrm{E}^{1}$ \\ Department of Anesthesiology and Reanimation, Faculty of Medicine, Ordu University, Ordu, Turkey. \\ nilaytasmd@gmail.com
}

\begin{abstract}
OBJECTIVE AND BACKGROUND: Acute acetaminophen (APAP) overdose has been shown to cause toxicity and the primary treatment medication is $\mathrm{N}$-acetylcysteine (NAC). Dexmedetomidine (DEX) is a sedative drug with known antioxidant properties. We researched whether DEX has an injury-reducing effect on toxicity. METHODS: Rats were divided into: Group I (control), Group II (APAP) Group III (NAC) Group IV (DEX) and Group $\vee(N A C+D E X)$. Histopathologic investigations of tissues were performed and glutathione peroxidase (GSH-Px), catalase (CAT), malondialdehyde (MDA), myeloperoxidase (MPO) and beta trace protein (PGD2S) levels were studied in blood samples.

RESULTS: DEX administration for hepatotoxicity and nephrotoxicity induced with APAP, caused a significant reduction in oxidative injury markers like MDA and MPO, a significant increase in GSH-Px level and a significant degree of amelioration in liver histopathologic scores.

CONCLUSION: DEX administration for APAP toxicity causes a reduction in oxidative injury biomarkers, increased antioxidant biomarker levels and significant reduction in liver histopathologic scores. The beneficial effect of DEX use for detection of toxicity induced by acute APAP overdose, was shown in this study for the first time (Tab. 5, Fig. 2, Ref. 41). Text in PDF www.elis.sk.

KEY WORDS: acetaminophen, dexmedetomidine, hepatotoxicity, nephrotoxicity.
\end{abstract}

\section{Introduction}

Acetaminophen (APAP: $N$-acetyl-p-aminophenol, paracetamol) is an analgesic antipyretic medication that is commonly used globally $(1,2,3)$. Therapeutic doses are safe, however toxic metabolites occurring during overdose cause severe organ toxicity (4). After oral intake a small portion of APAP (5-15\%) transforms into the toxic metabolite of $N$-acetyl-p-benzoquinoneimine (NAPQI). NAPQI causes mitochondrial oxidative injury and increased reactive oxygen species (ROS) development $(3,5,6)$. In overdose situations, NAPQI occurs at amounts that cannot be detoxified and this causes toxicity $(2,4)$. Currently, the best treatment known for APAP toxicity globally is $\mathrm{N}$-acetylcysteine (NAC) treatment protocol $(7,8)$. NAC is a GSH precursor with antioxidant effects $(9)$.

${ }^{1}$ Department of Anesthesiology and Reanimation, Faculty of Medicine, Ordu University, Ordu, Turkey, ${ }^{2}$ Department of Anesthesiology and Reanimation, Ministry of Health-Ordu University Training and Research Hospital, Ordu, Turkey, ${ }^{3}$ Department of Biochemistry, Faculty of Medicine, Ordu University, Ordu, Turkey, and ${ }^{4}$ Department of Histology and Embryology, Faculty of Medicine, Istanbul University, Istanbul, Turkey

Address for correspondence: N. Tas, Department of Anesthesiology and Reanimation, Faculty of Medicine, Ordu University, Postal zip code: 52200, Ordu, Turkey.

Phone: +90.452 .2252344 , Fax: +90.452 .2250190$

Acknowledgement: This study was supported by Ordu University Scientific Research Projects Coordination Department (Project Number: 2017; HD-1725).
It reduces mortality and morbidity if administered within the first 8 hours $(4,5,8)$. The worst clinical manifestation in APAP hepatotoxicity is fulminant liver failure. There are many studies about hepatotoxicity occurring with APAP in the literature. However, there are limited studies about the nephrotoxicity caused directly by APAP. Though much research reports that nephrotoxicity develops secondary to advanced stage hepatotoxicity, low numbers of studies have shown that nephrotoxicity may occur related to the direct effect of APAP (10). Many molecules have been trialed with the aim of preventing injury in APAP toxicity. Some of these have antioxidant properties and there are studies showing benefits $(2,4,6)$. Dexmedetomidine is a potent and specific $\alpha_{2}$ receptor agonist with sedative, anxiolytic and analgesic effects. $\alpha_{2}$ receptors are found in many organs like the liver, lungs, brain and kidneys. DEX is known to activate pro-survival kinases and endothelial nitric oxide synthesis, modifying oxidative and inflammatory responses (11). DEX has anti-inflammatory and antioxidant effects and is shown to reduce ischemia and reperfusion injury $(11,12)$. In this experimental research, we researched whether DEX had an injury-reducing effect on liver and renal toxicity experimentally induced by acute APAP toxicity in rats.

\section{Material and methods}

This experimental study was approved by Ordu University Animal Care and Ethics Committee (2017/17) and was supported by Ordu University Scientific Research Projects Coordination 
Tab. 1. Descriptive statistics and comparison results for GSH-Px $(\mathrm{pg} / \mathrm{mL})$.

\begin{tabular}{|c|c|c|c|c|c|c|}
\hline Groups & $\mathrm{n}$ & Mean & SEM & $\mathrm{SD}$ & Min. & Max. \\
\hline Control & 5 & $659.481 \mathrm{~A}$ & 69.908 & 156.320 & 433.774 & 805.472 \\
\hline APAP & 7 & $189.190 \mathrm{C}$ & 18.720 & 49.528 & 131.446 & 270.833 \\
\hline NAC & 6 & 481.968B & 50.985 & 124.888 & 336.746 & 701.591 \\
\hline DEX & 7 & 426.389B & 40.657 & 107.570 & 327.589 & 637.703 \\
\hline $\mathrm{NAC}+\mathrm{DEX}$ & 7 & $320.396 \mathrm{BC}$ & 15.103 & 39.960 & 270.833 & 387.482 \\
\hline $\mathrm{p}$ & \multicolumn{6}{|c|}{$0.000 * * *(\mathrm{~F}=18.47)$} \\
\hline
\end{tabular}

SEM - standard error of mean; SD - standard deviation; $* * *$ - statistically significant $(\mathrm{p}<0.001)$

Means that do not share a letter are significantly different $(\mathrm{p}<0.05)$

Tab. 2. Descriptive statistics and comparison results for CAT (KU/L).

\begin{tabular}{|c|c|c|c|c|c|c|}
\hline Groups & $\mathrm{n}$ & Mean & SEM & $\mathrm{SD}$ & Min. & Max. \\
\hline Control & 5 & $59.613 \mathrm{~A}$ & 1.716 & 3.838 & 53.480 & 63.368 \\
\hline Paracetamol & 7 & $50.655 \mathrm{C}$ & 1.208 & 3.196 & 45.840 & 55.278 \\
\hline NAC & 6 & $55.727 \mathrm{AB}$ & 1.101 & 2.697 & 51.233 & 58.873 \\
\hline DEX & 7 & $54.508 \mathrm{BC}$ & 0.876 & 2.317 & 51.233 & 57.975 \\
\hline $\mathrm{NAC}+\mathrm{DEX}$ & 7 & $52.261 \mathrm{BC}$ & 0.633 & 1.676 & 49.436 & 54.379 \\
\hline
\end{tabular}

p $0.000 * * *(\mathrm{~F}=9.04)$

SEM - standard error of mean; SD - standard deviation; $* * *$ - statistically significant $(\mathrm{p}<0.001)$

Means that do not share a letter are significantly different $(\mathrm{p}<0.05)$

Department (Project Number: 2017; HD-1725) Ordu, TURKEY. Animals could access standard diet and water and were housed in an environment with $40-60 \%$ humidity, $20-24{ }^{\circ} \mathrm{C}$ temperature and 12-hour light/dark cycles.

\section{Experimental procedure}

Rats were divided into 5 groups; animals in the control group received no medication. Animals in the APAP group were administered $1 \mathrm{~g} / \mathrm{kg}$ dose APAP (Parol tb. $500 \mathrm{mg}$, Atabay, Turkey) in 3 $\mathrm{ml}$ volume (diluted with $0.9 \% \mathrm{NaCl}$ ) via gavage. Animals in the NAC group received $400 \mathrm{mg} / \mathrm{kg}$ dose of NAC (Asist amp. 300 $\mathrm{mg} / 3 \mathrm{ml}$ Bilim, Turkey) via ip 1 hour after administration of the same APAP dose. Animals in the DEX group were administered $100 \mu \mathrm{g} / \mathrm{kg}$ DEX (Precedex flk $200 \mu \mathrm{g} / 2 \mathrm{ml}$, Hospira Inc, USA) via ip 1 hour after the same APAP dose. Animals in the NAC+DEX group were simultaneously administered $100 \mu \mathrm{g} / \mathrm{kg}$ dose DEX and $400 \mathrm{mg} / \mathrm{kg}$ dose NAC via ip after the same APAP dose. Twentyfour hours later animals had the same doses of ip NAC, DEX and $\mathrm{NAC}+\mathrm{DEX}$ repeated. Two hours after administration, the surgical field was opened and liver and kidney tissues were removed. Simultaneously, blood samples were taken by intracardiac puncture to examine GSH-Px, CAT, MDA, MPO and PGD2S levels.

\section{Tissue processing}

The tissues were fixed in $10 \%$ neutral buffered formalin, dehydrated and infiltrated with paraffin, and sectioned at about $5 \mu \mathrm{m}$ thickness. The renal general structure was demonstrated by staining with the hematoxylin and eosin. Glomerular area was determined in kidney sections stained with Masson trichrome procedure (HT15 kit; Sigma-Aldrich, St. Louis MO). Image analysis was used to quantitate glomerular area (ImagePro Plus 5.1; Media Cybernetics, Silver Spring, MD). The scoring of tissues for degeneration, type of the degeneration and infiltration of inflammatory cells were a modification of Knodell method $(13,14,15)$. The glomerular area was determined in Masson trichrome-stained sections by calculat- ing the mean of 5 glomeruli per animal at $\mathrm{x} 400$ magnification in the cortical zone $(16,17)$.

\section{Biochemical analysis procedure}

Blood samples were obtained for measuring the activities of GSH-Px, CAT, MPO, PGD2S and MDA levels. Then, they were also centrifuged and stored at $-70{ }^{\circ} \mathrm{C}$ until analyzed. Serum GSH-Px and PGD2S activities were measured using commercial kits (Elabscience Biotechnology Co., Ltd., USA) based EnzymeLinked Immunosorbent Assay (ELISA) methods. Serum CAT and MPO activities, and also MDA levels were measured by colorimetric method (respectively) $(18,19,20)$.

\section{Statistical analysis}

The Kolmogorov-Smirnov test was used to check normal distribution of data, with the Levene test performed to check homogeneity in group variance. One-way analysis of variance (ANOVA) was used to analyze data. After variance analysis, differing groups were determined with Tukey multiple comparison test and results are represented by letters. Significance level $(\alpha)$ $5 \%$ was used for calculations and interpretation of results. All calculations were performed with SPSS v25 (IBM Inc., Chicago, IL, USA) statistical program.

\section{Results}

\section{Biochemical evaluation}

The control group had significantly high mean GSH-Px compared to the other groups $(\mathrm{p}<0.05)$. There was no significant difference between the NAC, DEX and NAC+DEX groups ( $p>$ $0.05)$. The APAP group had statistically significantly lower mean GSH-Px compared to the NAC and DEX groups $(\mathrm{p}<0.05)($ Tab. 1). According to analysis in terms of CAT, there were significant differences between APAP and NAC groups ( $p<0.05)$ (Tab. 2), with no significant differences identified in terms of APAP, DEX 
Tab. 3. Descriptive statistics and comparison results for MDA $(\mu \mathrm{mol} / \mathrm{L})$.

\begin{tabular}{|c|c|c|c|c|c|c|}
\hline Groups & $\mathrm{n}$ & Mean & SEM & SD & Min. & Max. \\
\hline Control & 5 & $0.323 \mathrm{~B}$ & 0.044 & 0.097 & 0.221 & 0.446 \\
\hline Paracetamol & 7 & $0.778 \mathrm{~A}$ & 0.115 & 0.304 & 0.443 & 1.400 \\
\hline NAC & 6 & $0.321 \mathrm{~B}$ & 0.023 & 0.057 & 0.237 & 0.396 \\
\hline DEX & 7 & $0.273 \mathrm{~B}$ & 0.032 & 0.084 & 0.165 & 0.382 \\
\hline $\mathrm{NAC}+\mathrm{DEX}$ & 7 & $0.400 \mathrm{~B}$ & 0.050 & 0.132 & 0.225 & 0.589 \\
\hline $\mathrm{p}$ & \multicolumn{6}{|c|}{$0.000 * * *(\mathrm{~F}=10.34)$} \\
\hline
\end{tabular}

SEM - standard error of mean; SD - standard deviation; $* * *$ - statistically significant $(\mathrm{p}<0.001)$

Means that do not share a letter are significantly different $(\mathrm{p}<0.05)$

Tab. 4. Descriptive statistics and comparison results for MPO (U/L).

\begin{tabular}{|c|c|c|c|c|c|c|}
\hline Groups & $\mathrm{n}$ & Mean & SEM & SD & Min. & Max. \\
\hline Control & 5 & $13.619 \mathrm{C}$ & 1.385 & 3.097 & 10.772 & 18.594 \\
\hline Paracetamol & 7 & $31.083 \mathrm{~A}$ & 3.201 & 8.468 & 18.594 & 43.175 \\
\hline $\mathrm{NAC}$ & 6 & $19.086 \mathrm{BC}$ & 2.583 & 6.327 & 11.541 & 28.854 \\
\hline DEX & 7 & $21.544 \mathrm{BC}$ & 0.968 & 2.561 & 19.236 & 25.648 \\
\hline $\mathrm{NAC}+\mathrm{DEX}$ & 7 & $25.849 \mathrm{AB}$ & 2.267 & 5.999 & 20.518 & 35.266 \\
\hline
\end{tabular}

SEM - standard error of mean; SD - standard deviation; *** - statistically significant $(\mathrm{p}<0.001)$

Means that do not share a letter are significantly different $(\mathrm{p}<0.05)$

Tab. 5. Descriptive statistics and comparison results for Beta trace (PGD2S) (ng/mL).

\begin{tabular}{llllll}
\hline Groups & $\mathrm{n}$ & Mean & SEM & SD & Max. \\
\hline Control & 5 & 3.279 & 0.750 & 1.984 & 0.621 \\
Paracetamol & 7 & 3.770 & 0.796 & 1.779 & 1.769 \\
NAC & 6 & 2.013 & 0.242 & 0.593 & 0.835 \\
DEX & 7 & 2.705 & 0.353 & 0.935 & 2.626 \\
NAC+DEX & 7 & 2.000 & 0.339 & 0.897 & 1.769 \\
p & & & $0.121(\mathrm{~F}=2.01)$ & 3.327 & 0.766 \\
\hline
\end{tabular}

SEM - standard error of mean; SD - standard deviation
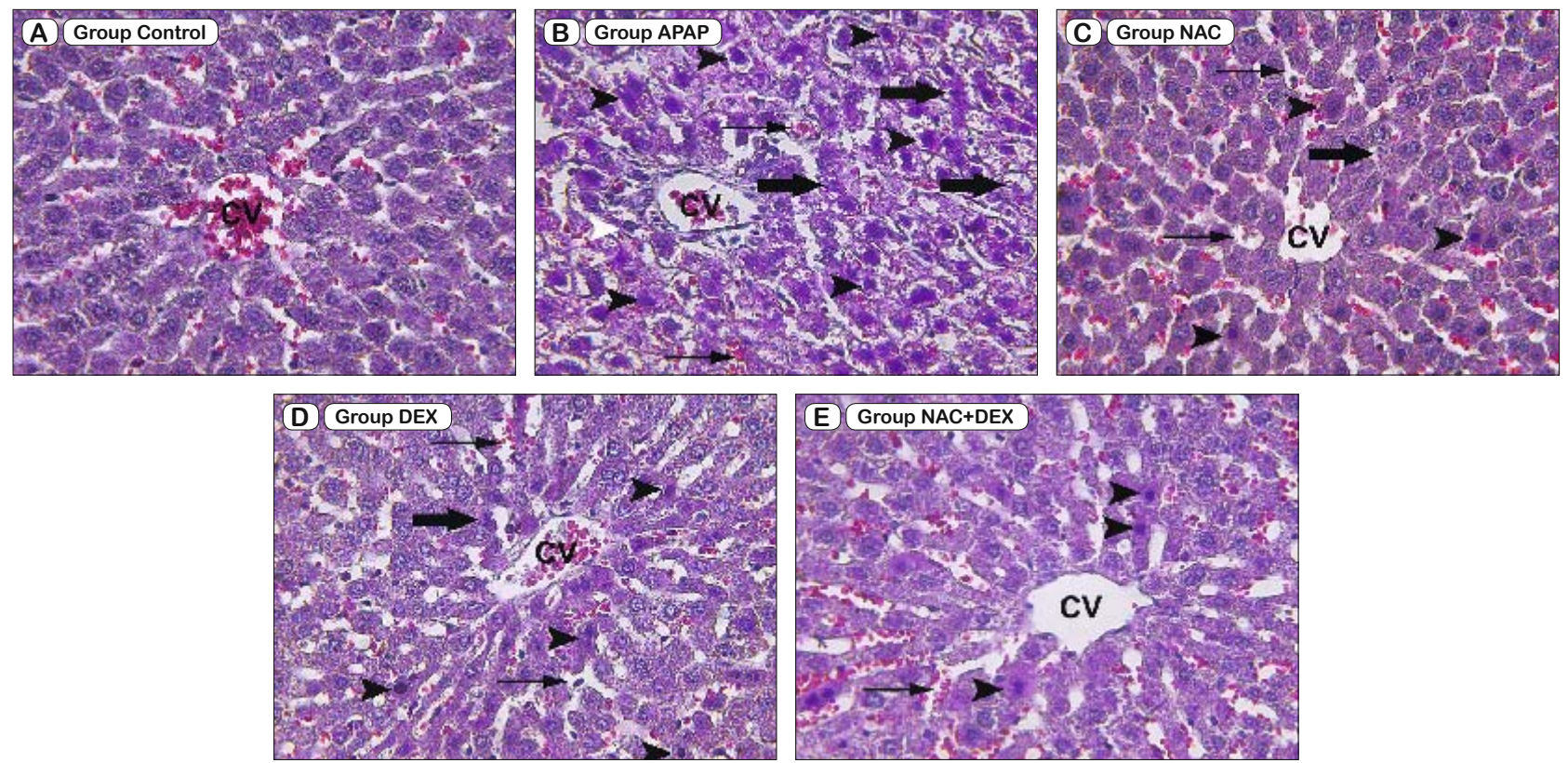

Fig. 1. (A) Hepatic histology of the Control group showing normal hepatocytes and central vein (CV). (B) Group APAP showing enlarged hepatocytes with vacuolated and enlarged cytoplasm and small pyknotic nuclei (arrowheads) or fragmented nuclei (thick arrow) and compressing the sinusoids (thin arrows). (C) Group NAC have a few hepatocytes with dark acidophilic cytoplasm (arrowheads) and pyknotic nuclei (thick arrow) and a little dilated sinusoids (arrows). (D) Group DEX have most hepatocytes with dark stained acidophilic cytoplasm (arrowheads) and pyknotic nuclei (thick arrow). Some sinusoids are markedly dilated (arrows). (E) Group NAC+DEX showing a few hepatocytes with dark acidophilic cytoplasm (arrowheads) and pyknotic nuclei (thick arrow) and a little dilated sinusoids (arrows). H\&E, Mag x 400. 

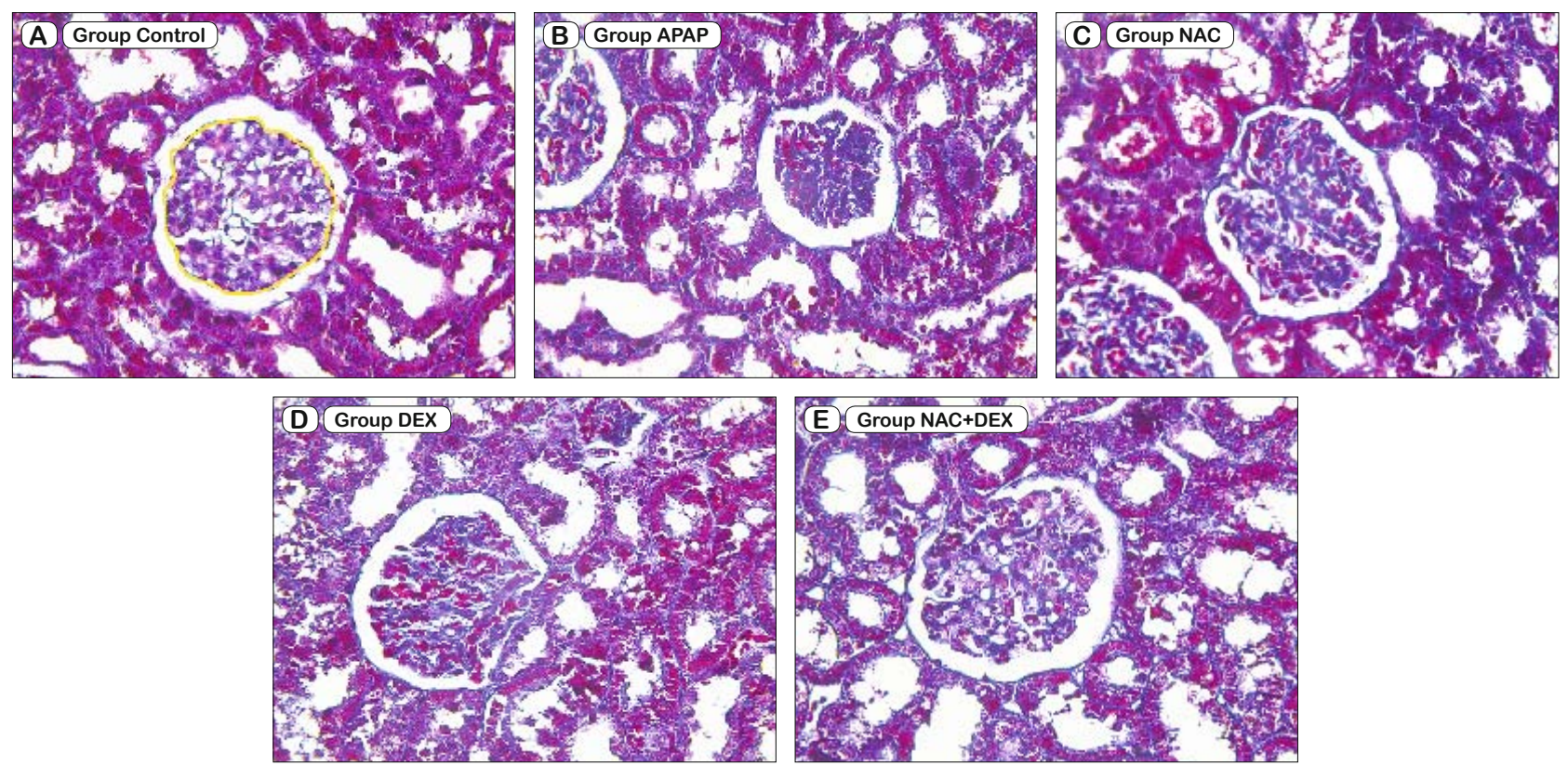

Fig. 2. (A) Representative an example of the glomerular area measurement of a normal glomeruli is shown in Control group. The glomerular area was defined as the area of the outer capillary loops of the tuft (inner area of yellow outline). (B) Group APAP; in sclerotic glomeruli, marked and complete loss of glomerular capillaries was noted with massive accumulation of extracellular matrix in glomeruli. (C) Group NAC; showing the glomeruli with minimal glomerular abnormalities. (D) Group DEX; showing the glomeruli with sclerotic glomeruli. (E) Group NAC+DEX; glomeruli was noted with minimal extracellular matrix accumulation. Masson trichrome stain, Mag x400.

and NAC+DEX groups ( $\mathrm{p}>0.05)$ (Tab. 2). The APAP group was identified to have a significant increase in mean MDA compared to the control group, with NAC, DEX and NAC+DEX groups all having significant reductions in MDA levels compared to the APAP group $(\mathrm{p}<0.001)$ (Tab. 3). Mean MPO in the APAP group was significantly higher, with NAC and DEX groups identified to be significantly lower compared to the APAP group $(\mathrm{p}<0.001)$ (Tab. 4). There was no significant difference found between the NAC+DEX and APAP groups $(p>0.05)($ Tab. 4). The results of variance analysis for PGD2S did not find the differences between the groups were statistically significant $(p>0.05)$ (Tab. 5).

\section{Histological evaluation}

The Control group exhibited normal hepatocytes (Fig. 1A). In the liver of the Group APAP, disruption of abnormal architecture of necrotic and apoptotic hepatocytes with dark acidophilic stained cytoplasm and fragmented nuclei was observed. Most hepatocytes were vacuolated and degenerated in the Group APAP. The disappeared sinusoids because of the sinusoidal cell accumulation were not clear (Fig. 1B). The number of hepatocytes with dark acidophilic cytoplasm and pyknotic nuclei were decreased in the Group DEX in comparison to Group APAP with a little effect on dilated sinusoids (Fig. 1D). In the Group NAC and NAC+DEX, there was a significant decrease in hepatocytes degeneration and dilated sinusoids values of liver tissue, compared to both Group APAP and Group DEX (Figs. 1C and 1E). In the Control group, the glomerules had normal morphology (Fig. 2A). When compared to the control group animals, a significant increase in histological score was recorded from Group APAP and Group DEX rats, indicating significant tubular injury (Figs 2B and 2D). In the Group APAP and Group DEX, tubules become atrophied and dilated and appeared both apoptotic and necrotic . There was a large number of infiltrating polymorphonuclear leukocytes in renal cortex obtained from Group APAP and Group DEX rats. Compared with control group, mean glomerular area was significantly reduced in the Group APAP and DEX $(p<0.0001)$ In the Group APAP and Group DEX, the glomerular area decreased with narrowing glomerular capillaries and accumulation of glomerular extracellular matrix in glomerulus in the development of glomerular sclerosis. The histological scores of the tubular injury were significantly reduced in the Group NAC and Group NAC+DEX when compared to values obtained from Group APAP and Group DEX rats $(\mathrm{p}<0.0001$, Figure $2 \mathrm{C}$ and $2 \mathrm{E})$. There was only a minimal number of polymorphonuclear leukocytes in renal cortex obtained from Group NAC and Group NAC+DEX rats. The degree of glomerulosclerosis was significantly reduced in the Group NAC and Group NAC+DEX.

\section{Discussion}

Due to acute APAP intoxication, each year tens of thousands of people are known to be admitted to hospital. For the first time at the end of the 1960s, APAP overdose was identified to cause severe liver failure, renal failure and death (21). Oxidative stress in the APAP toxicity has many characteristics such as lipid peroxidation and ROS levels increase within the first 24 hours after toxic intake 


\section{0-276}

$(4,22)$. Increased ROS levels decrease the levels of GSH, the main intracellular defense molecule preventing oxidative stress in cells, and reduce CAT enzyme function activity. GSH-Px is responsible for reducing $\mathrm{H}_{2} \mathrm{O}_{2}$ and lipid peroxides and is known to have a protective role in APAP toxicity $(22,23)$. In our research, analysis for GSH-Px found significant differences between the groups. NAC and DEX groups had statistically significantly higher mean GSH-Px compared to the APAP group, while there were no significant differences identified between NAC, DEX and NAC+DEX groups. CAT enzyme activity was significantly reduced in the liver 24 hours after APAP administration and antioxidant treatment increased CAT activity (4). In our study, APAP administration caused a reduction in CAT levels, while administration of NAC alone caused a significant increase in CAT levels. Administration of DEX alone and NAC+DEX together did not cause a significant increase in CAT activity. As known, the increase in ROS causes production of MDA, the final product of lipid peroxidation and a marker of oxidative stress (4). In our study, the MDA level was significantly increased in the APAP group and significant levels of reduction were identified in the NAC, DEX and NAC+DEX treatment groups compared to the APAP group. However, there was no significant difference identified between the treatment groups. Experimental research by Kuvandik et al reported significantly high MDA levels in the APAP group and the same research stated that groups administered erdosteine treatment had this elevation significantly prevented (22). Research by Aycan et al showed significant increase in MDA levels in the APAP toxicity group with a significant fall in MDA levels in the group treated with thymoquinone (24). MPO is a proinflammatory protein and it is a beneficial risk determinant in many clinical situations including oxidative stress (25). An important mechanism in APAP toxicity is thought to be oxidative stress including MPO utilization (26). Research by Uchida et al reported high dose of APAP causing a significant increase in MPO levels, and they observed the use of the natural bioactive antioxidant material of citral caused a significant fall in MPO levels (27). Dwivedi et al in a study using the herbal formulation of livartho observed significant increases in MPO levels in rats exposed to APAP, while there was a significant fall in MPO level close to the control group in the group treated with livartho (28). In our research MPO level in the group administered APAP overdose was observed to be significantly elevated compared to the control group. The treatment groups of NAC alone and DEX alone were identified to have significant reduction in MPO levels compared to the APAP group. This difference was not significant in the group administered NAC+DEX together.

APAP toxicity causes serious histopathologic changes in the form of centrilobular hepatic necrosis and inflammatory cell infiltration (6). In our research, the liver histopathologic score in the APAP group was significantly increased compared to the control group. All of the treatment groups of NAC, DEX and NAC+DEX were identified to have significant decrease in this score. This reduction was more significant in the NAC and NAC+DEX groups. In ischemia-reperfusion injury and oxidative stress studies with DEX it has been reported that DEX has a histopathological improvement effect and regression effect in damaged tissues of dif- ferent organs $(29,30)$. In an experimental study, Sancaktar et al reported that there was a significant decrease in renal apoptotic index in the DEX group compared to the I/R damage group (29). Again Chen et al showed that use of DEX reduces apoptosis in cardiopulmonary bypass related neuronal apoptosis (31). In another experimental study, Cakir et al reported that DEX has been shown to reduce histological damage on large tubular necrosis and glomerular damage secondary to I/R injury (32). In our study, similar to the literature, liver histopathological score decreased with DEX and apoptotic and degenerated cells were significantly decreased compared to APAP group too. As known, therapeutic doses of APAP are metabolized in the liver and water-soluble end-products are excreted through the kidneys. APAP overdose increases lipid peroxidation in renal tissue (33). In spite of intense explanation of mechanisms related to hepatotoxicity, molecular explanations about apoptosis of tubular cells and acute renal injury are encountered less in the literature (34). Loss of membrane asymmetry in tubular cells and stress are thought to play a role in nephrotoxicity induced by APAP. Additionally, acute APAP overdose is reported to be possibly associated with local hemodynamic factors in the kidney, COX inhibition similar to NSAIDs and vasoconstriction occurring in the early stage $(34,35)$. In our study, histopathologic investigation of the APAP group recorded significant tubular injury and significant increases in histologic scores. In the NAC and NAC+DEX treatment groups, there were significant regressions in nephrotoxic injury scores; however, DEX alone was not observed to cause amelioration in this score. Acute kidney injury is a complex and dynamic process (36). PGD2S is a new determinant of glomerular filtration rate. The use of PGD2S as a biomarker in acute renal injury is currently increasing (37). It is known that when the glomerular filtration rate reduces, serum PGD2S level and the fractional clearance of PGD2S increases with urinary PGD2S (38). According to analysis, there was no significant outcome obtained between the groups in terms of PGD2S levels. Because of the fractional clearance of PGD2S and no urinary PGD2S observed in our study, it is possible to say that there is a limitation in this direction.

When the literature is examined, it is possible to see many molecules with therapeutic or preventive properties used in research about APAP toxicity $(2,4,6)$. Dexmedetomidine is an effective agent used for sedation in intensive care and anesthesia administration with increasing use as an adjuvant $(39,40,41)$. With antioxidant properties proven in many researches, DEX is understood not to have been used in experimental research related to APAP toxicity in the literature. As a result, our study carries the feature of being the first on this topic. In our study, administration of DEX for APAP toxicity was identified to cause a significant reduction in oxidative injury marker MPO and MDA levels. The effect on CAT was not accepted as significant, but DEX administration was determined to cause a significant increase in the antioxidant biomarker of GSH-Px. When examined histopathologically, the DEX group had significantly reduced dark acidophilic cytoplasm and pyknotic nuclei numbers compared to the APAP group. When assessed in terms of renal injury, DEX treatment was not observed to cause an improvement as significant as NAC and NAC+DEX groups in 
terms of tubular injury histologic scores. Though the histopathologic improvement in terms of the liver was clear in the DEX group, there was no similar amelioration in kidney tubular injury histologic scores which leads to the consideration that the renal injury mechanism may be different to the liver injury mechanism $(34,35)$. It is possible to find clinical and experimental studies with different doses of DEX in the literature $(39,40,41)$. Behmenburg et al used low (0.1-1 nM) and high (3-30 nM) doses of DEX during ischemia and reperfusion of isolated rat hearts and reported high-dose DEX ensured better regression of infarct dimensions compared to low doses (41). Wan et al administered $0.25,0.5$ and $1 \mu \mathrm{g} / \mathrm{kg}$ doses of dexmedetomidine to patients undergoing laparoscopic surgery with general anesthesia and examined CRP, TNF- $\alpha$ and IL-10 levels. Compared to the $0.25 \mu \mathrm{g} / \mathrm{kg}$ dose, patients receiving 0.5 and $1 \mu \mathrm{g} / \mathrm{kg}$ had lower CRP and TNF- $\alpha$ levels, while the IL-10 level was observed to be highest in the $1 \mu \mathrm{g} / \mathrm{kg}$ group (40). Based on this result, this may be a new research topic in terms of the effect of DEX on APAP toxicity and there is a need for new studies involving different dose applications.

According to the results of our research, DEX administration for hepatotoxicity and nephrotoxicity induced with APAP caused a significant reduction in oxidative injury markers like MDA and MPO, a significant increase in GSH-Px level and a significant degree of amelioration in liver histopathologic scores. Though many antioxidant molecules have been studied for lethal hepatotoxicity and nephrotoxicity developing and link to acute APAP overdose, the effects of DEX were investigated for the first time in this study. Effectively used as a sedative analgesic in modern anesthesia and intensive care practice, DEX appears to be frequently studied in different experimental oxidative injury manifestation. Our research leads to the consideration that DEX may have positive effects during use with treatment aims for the oxidative injury manifestation induced by acute APAP overdose.

\section{Learning points:}

- Oxidative stress in the APAP toxicity has many characteristics such as lipid peroxidation, mitochondrial injury and ROS levels increase.

- In oxidative stress studies with DEX it has been reported that DEX is connected with a histopathological improvement and regression in damaged tissues of different organs.

- DEX administration for APAP toxicity causes reduction in oxidative injury biomarkers, increased antioxidant biomarker levels and significant reduction in liver histopathologic scores.

\section{References}

1. Levine M, O'Connor AD, Padilla-Jones A, Gerkin RD. Comparison of Prothrombin Time and Aspartate Aminotransferase in Predicting Hepatotoxicity After Acetaminophen Overdose. J Med Toxicol 2016; 12 (1): 100-106.

2. Ishii I, Kamata S, Hagiya Y, Abiko Y, Kasahara T, Kumagai Y. Protective effects of hydrogen sulfide anions against acetaminophen-induced hepatotoxicity in mice. J Toxicol Sci 2015; 40 (6): 837-841.
3. Ghanem CI, Pérez MJ, Manautou JE, Mottino AD. Acetaminophene from liver to brain: New insights into drug pharmacological action and toxicity. Pharmacol Res 2016; 109: 119-131.

4. Nikravesh H, Khodayar MJ, Mahdavinia M, Mansouri E, Zeidooni L, Dehbashi F. Protective Effect of Gemfibrozil on Hepatotoxicity Induced by Acetaminophen in Mice: the Importance of Oxidative Stress Suppression. Adv Pharm Bull. 2018; 8 (2): 331-339.

5. Janssen J, Singh-Saluja S. How much did you take? Reviewing acetaminophen toxicity. Can Fam Physician. 2015; 61 (4): 347-349.

6. Mazraati P, Minaiyan M. Hepatoprotective Effect of Metadoxine on Acetaminophen-induced Liver Toxicity in Mice. Adv Biomed Res 2018; 24 (7): 67.

7. Chiew AL, Gluud C, Brok J, Buckley NA. Interventions for paracetamol (acetaminophen) overdose. Cochrane Database Syst Rev 2018; 23 (2): CD003328.

8. Papazoglu C, Ang JR, Mandel M, Basak P, Jesmajian S. Acetaminophen overdose associated with double serum concentration peaks. J Community Hosp Intern Med Perspect 201511; 5 (6): 29589.

9. Anouar T. The role of $\mathrm{N}$-acetylcysteine in the treatment of non-acetaminophen acute liver failure. Saudi J Gastroenterol 2017; 23 (3): 131-132.

10. Loh CS, Ponampalam R. Nephrotoxicity associated with acute paracetamol overdose: a case report and review of the literature. Hong Kong J Emerg Med 2006; 13 (2): 105-110.

11. Weerink MAS, Struys MMRF, Hannivoort LN, Barends CRM, Absalom AR, Colin P. Clinical Pharmacokinetics and Pharmacodynamics of Dexmedetomidine. Clin Pharmacokinet 2017; 56 (8): 893-813.

12. Zhou Y, Zhou X, Zhou W, Pang Q, Wang Z. The protective effect of dexmedetomidine in a rat ex vivo lung model of ischemia-reperfusion injury. Acta Cir Bras 2018; 33 (1): 1-13.

13. Crosse K, Umeadi OG, Anania FA et al. Racial differences in liver inflammation and fibrosis related to chronic hepatitis C. Clin Gastroenterol Hepatol 2004; 2 (6): 463-468.

14. French SW, Miyamoto K, Ohta Y, Geoffrion Y. Pathogenesis of experimental alcoholic liver disease in the rat. Methods Achiev Exp Pathol 1988; 13: 181-207.

15. Knodell RG, Ishak KG, Black WC et al. Formulation and application of a numerical scoring system for assessing histological activity in asymptomatic chronic active hepatitis. Hepatology 1981; 1 (5): 431-435.

16. Thiemermann C, Patel NS, Kvale EO et al. High density lipoprotein (HDL) reduces renal ischemia/reperfusion injury. J Am Soc Nephrol 2003; 14 (7); 1833-1843.

17. Velez JC, Janech MG, Hicks MP et al. Lack of renoprotective effect of chronic intravenous angiotensin- (1-7) or angiotensin- (2-10) in a rat model of focal segmental glomerulosclerosis. PLoS One 2014; 9 (10): e110083.

18. Goth $\mathbf{L}$. A siple method for determation of serum catalase activation and revision of reference range. Clin Chim Acta 1992; 196 (2-3): 143-151.

19. Bradley PP, Priebat DA, Christensen RD, Rothstein G. Measurement of cutaneous inflammation: estimation of neutrophil content with an enzyme marker. J Invest Dermatol 1982; 78 (3): 206-209.

20. Wasowicz W, Neve J, Peretz A. Optimized steps in fluorometric determination of thiobarbituric acit-reactive substances in serum: importance of extraction Ph and influence of sample preservation and stroge, Clin Chem 1993; 39 (12): 2522-2526. 


\section{0-276}

21. Davidson DG, Eastham WN. Acute liver necrosis following overdose of paracetamol. Br Med J 1966; 27; 2 (5512): 497-499.

22. Kuvandik G, Duru M, Nacar A et al. Effects of erdosteine on acetaminophen-induced hepatotoxicity in rats. Toxicol Pathol. 2008; 36 (5): 714-719.

23. Birben E, Sahiner UM, Sackesen C, Erzurum S, Kalayci O. Oxidative stress and antioxidant defense. World Allergy Organ J 2012; 5 (1): 9-19.

24. Aycan IÖ, Tüfek A, Tokgöz O, Evliyaoğlu O, Firat U, Kavak Gö, Turgut H, Yüksel MU. Thymoquinone treatment against acetaminophen-induced hepatotoxicity in rats. Int J Surg 2014; 12 (3): 213-218.

25. Loria V, Dato I, Graziani F, Biasucci LM. Myeloperoxidase: A New Biomarker of Inflammation in Ischemic Heart Disease and Acute Coronary Syndromes. Mediators Inflamm 2008; 2008: 135625.

26. Hinson JA, Roberts DW, James LP. Mechanisms of Acetaminopheninduced Liver Necrosis. Handb Exp Pharmacol 2010; 196: 369-405.

27. Uchida NS, Silva-Filho SE, Cardia GFE et al. Hepatoprotective Effect of Citral on Acetaminophen-induced Liver Toxicity in Mice. Evid Based Complement Alternat Med 2017; 2017: 1796209.

28. Dwivedi VK, Mishra J, Shrivastava A. Efficacy Study of Livartho against Paracetamol Induced Hepatotoxicity in Adult Sprague Dawley Rats. J Drug Metab Toxicol 2015; 5 (6): 1-7

29. Sancaktar NC, Altinbaş A, Cekic B. Protective Role of Dexmedetomidine on Ileum and Kidney Damage Caused by Mesenchymal Ischaemia in Rats. Turk J Anaesthesiol Reanim 2018; 46 (6): 470-477.

30. Karahan MA, Yalcin S, Aydogan $\mathbf{H}$ et al. Curcumin and dexmedetomidine prevents oxidative stress and renal injury in hind limbischemia/ reperfusion injury in a rat model. Ren Fail 2016; 38 (5): 693-698.

31. Chen Y, Zhang X, Zhang B, He G, Zhou L, Xie Y. Dexmedetomidine reduces the neuronal apoptosis related to cardiopulmonary bypass by inhibiting activation of the JAK2-STAT3 pathway. Drug Des Devel Ther 2017; 26 (11): 2787-2799.
32. Cakir M, Polat A, Tekin S et al. The effect of dexmedetomidine against oxidative and tubular damage induced by renal ischemia reperfusion in rats. Ren Failure 2015; 37 (4): 704-708.

33. Dokumacioglu E, Iskender H. Acetaminophen-Induced Nephrotoxicity and Cystatin-C. JOJ uro \& nephron 2017; 1 (5): 1-2. JOJUN. MS.ID.555571.

34. Lorz C, Justo P, Sanz A, Subirá D, Egido J, Ortiz A. Paracetamolinduced renal tubular injury: a role for ER stress. J Am Soc Nephrol 2004; 15 (2): 380-389.

35. Pakravan N, Bateman DN, Goddard J. Effect of acute paracetamol overdose on changes in serum and urine electrolytes. Br J Clin Pharmacol 2007; 64 (6): 824-832.

36. de Carvalho AL, Vital RB, Kakuda CM et al. Dexmedetomidine on renal ischemia-reperfusion injury in rats: assessment by means of NGALand histology. Ren Fail. 2015 Apr; 37 (3): 526-530.

37. Chen HH. $\beta$-trace protein versus cystatin $C$ : which is a better surrogate marker of renal function versusprognostic indicator in cardiovascular diseases? J Am Coll Cardiol 2011; 57 (7): 859-860.

38. Donadio C, Bozzoli L. Urinary $\beta$-trace protein: A unique biomarker to screen early glomerular filtration rate impairment. Medicine (Baltimore) 2016; 95 (49): e5553

39. Akpinar H, Akpinar O. The effects of dexmedetomidine on biomarkers of oxidative stress and antioxidants in kidney. Bratisl Med J 2018; 119 (8): 476-480.

40. Wan Z, Wang J, Cao H, Wu L. Effects of different doses of dexmedetomidine on analgesic efficacy and inflammatory cytokines in patients undergoing laparoscopic surgery. Exp Ther Med 2018; 16 (3): 1743-746. doi: $10.3892 /$ etm.2018.6355.

41. Behmenburg F, Pickert E, Mathes A, Heinen A, Hollmann MW, Huhn R, Berger MM. The Cardioprotective Effect Of Dexmedetomidine in Rats Is Dose-Dependent and Mediated by BKCa Channels. J Cardiovasc Pharmacol 2017; 69 (4): 228-235. doi:10.1097/FJC. 0000000000000466.

Received January 11, 2019. Accepted February 14, 2019. 